\title{
Engineering Research and Development of Magnetically Levitated High-Temperature Superconducting Coil System for Mini-RT Project
}

\author{
Nagato Yanagi, Junji Morikawa, Toshiyuki Mito, Yuichi Ogawa, Shinji Hamaguchi, Hirotaka Chikaraishi, \\ Masataka Iwakuma, Toshio Uede, Ikuo Itoh, Shinichi Nose, and Shigeo Fukui
}

\begin{abstract}
A magnetically levitated superconducting coil system is being developed using high temperature superconductors for examining a new magnetic confinement of high-beta plasmas. A miniature double-pancake coil was fabricated with a Bi-2223 Ag-sheathed tape for the purpose of developing a floating control using laser displacement gauges. The coil was inductively excited with liquid nitrogen cooling and successfully levitated in the air. A persistent current switch is also being developed with a Bi-2223 Ag-0.3wt\%Mn-sheathed tape, and a prototype model was successfully tested.
\end{abstract}

Index Terms-Bi-2223, high temperature superconductor, magnetic levitation, persistent current switch, plasma confinement.

\section{INTRODUCTION}

A N ADVANCED confinement scenario for high-beta nonneutral plasmas with a new relaxation process has been proposed by Mahajan and Yoshida [1]. The new magnetic configuration primarily incorporates a dipole magnetic field which is generated by a magnetically levitated superconducting coil. In order to experimentally examine this confinement scenario, a series of devices is being proposed at the High Temperature Plasma Center of the University of Tokyo [2]. The present goal is to build a middle-sized device, S-RT (here, RT denotes "Ring Trap") with a superconducting coil that has a diameter of 800 $\mathrm{mm}$. Although the confinement physics is rather different, this machine will look similar to the Levitated Dipole Experiment (LDX) which is being constructed under collaboration between Massachusetts Institute of Technology and Columbia University [3].

Before going to S-RT, it has been decided to build a smaller device to carry out both the proof-of-principle experiment for plasma confinement and to develop necessary engineering technologies to realize a superconducting floating device. For this purpose, Mini-RT is now being fabricated with a superconducting floating coil having a diameter of $300 \mathrm{~mm}$. Since the

Manuscript received September 24, 2001. This work was supported in part by the Japanese Ministry of Science and Education under Grant 12780368.

N. Yanagi, T. Mito, S. Hamaguchi, and H. Chikaraishi are with the National Institute for Fusion Science, Toki, Gifu 509-5292 Japan (e-mail: yanagi@nifs.ac.jp).

J. Morikawa and Y. Ogawa are with the University of Tokyo, Bunkyo-ku, Tokyo 113-8656, Japan.

M. Iwakuma is with Kyushu University, Fukuoka 812-8581, Japan.

T. Uede, I. Itoh, and S. Nose are with Fuji Electric Co., Kawasaki-ku, Kawasaki 210-9530, Japan.

S. Fukui is with Cryovac Co., Nishi-yodogawa-ku, Osaka 555-1103, Japan. Publisher Item Identifier S 1051-8223(02)03862-9.
TABLE I

MAJOR SPECIFICATIONS OF THE FB-RT FLOATING COIL

\begin{tabular}{lc}
\hline Inner / Outer Diameter & $77 / 94.5 \mathrm{~mm}$ \\
Number of Turns & 44 \\
Winding Method & Double Pancake \\
Insulation between Turns & Kapton Tape \\
Inductance & $0.272 \mathrm{mH}$ \\
Cable Type & Ag-sheathed Bi-2223 \\
Cable Supplier & (reinforced by S.S. foils) \\
Cable Width / Thickness & American Superconductor \\
Cable Length & $4.1 / 0.3 \mathrm{~mm}$ \\
Nominal Current & $12.0 \mathrm{~m}$ \\
Critical Current (77K, self-field) & $47 \mathrm{~A}$ \\
Critical Current (as a coil) & $118 \mathrm{~A}$ \\
Joint Resistance & $59 \mathrm{~A}$ \\
Coolant & $84 \mathrm{n} \Omega$ \\
Weight (including LN 2 ) & Liquid Nitrogen \\
\hline
\end{tabular}

floating coil has no refrigeration power during magnetic levitation, it is of primary importance to have high heat capacity. For Mini-RT, eight hours of magnetic levitation is required to effectively carry out plasma experiments. In order to satisfy this requirement, using high temperature superconducting (HTS) conductors for the winding seems very attractive, because HTS can be used at high temperature where the heat capacity of the coil becomes considerably higher than that for low temperature case.

\section{FB-RT EXPERIMENT}

\section{A. Fabrication of a Miniature Floating Coil for FB-RT}

Magnetic levitation of a superconducting coil is possible by having a real-time feedback control of the floating position. This control is realized both by precisely measuring the floating position, which is performed using laser displacement gauges in our system, and by rapidly and minutely changing the current in the levitating coil (which is installed above the floating coil) depending on the floating position.

The development of the control system for magnetic levitation was initiated by using a permanent magnet instead of a superconducting coil. However, after the basic feedback control circuit was successfully constructed, there was a need to actually test this system with a superconducting coil before it will be applied to Mini-RT. For this purpose, it was decided to prepare a small device, FB-RT, with a miniature floating coil [4]. The 


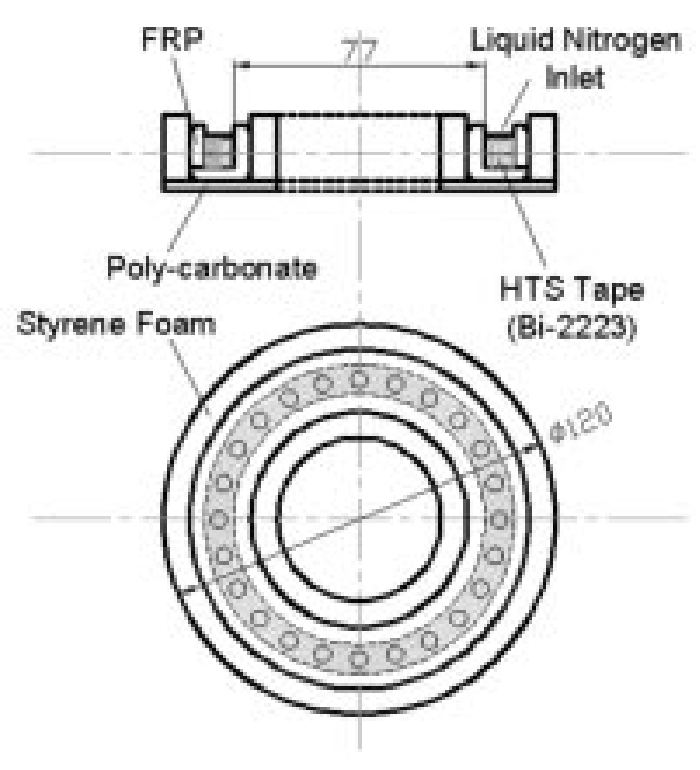

Fig. 1. Schematic illustrations of the FB-RT floating coil. Unit: mm.

side and plan views of the coil are illustrated in Fig. 1, and the major specifications are listed in Table I. For having an easy access for laser beams, the magnetic levitation is performed in the air, and an HTS superconducting winding is the only solution to meet this demand.

The miniature floating coil consists of a double-pancake winding of 44 turns, and the average radius is $42 \mathrm{~mm}$. A Bi-2223 Ag-sheathed tape (reinforced with stainless steel foils) [5] is used with a total length of $12 \mathrm{~m}$. A small winding machine was prepared, and the HTS tape was wound into a fiber-reinforced plastic (FRP) bobbin with an inner diameter of $77 \mathrm{~mm}$. The inter-turn insulation was given by a Kapton tape, and Epoxy glue was used between turns to ensure mechanical rigidity. The two ends of the tape were jointed at the outermost turn of the coil over the length of $\sim 150 \mathrm{~mm}$ using solder. The FRP bobbin has a groove to store liquid nitrogen, and the coil is thermally insulated by styrene-foam.

One of the most important issues for designing this miniature coil was to evaluate the expected critical current of the superconductor used for the winding. We assume that the critical current density is locally determined in the cross-section of the tape depending on the local magnetic field, and the current density is self-consistently calculated. Fig. 2 shows the profiles of the expected current density and the magnetic field, which correspond to the critical current condition of $59.2 \mathrm{~A}$ at $77 \mathrm{~K}$. Here, the critical current of a single conductor is given as $118 \mathrm{~A}$ at 77 $\mathrm{K}$, self-field, with a criterion for voltage generation of $1 \mu \mathrm{V} / \mathrm{cm}$.

\section{B. Excitation and Magnetic Levitation Experiments}

The excitation test of the miniature HTS coil has been conducted using a "field cooling" method, i.e., by cooling the coil with liquid nitrogen under the external magnetic field. Fig. 3 shows a waveform of the generated magnetic field measured by a Hall probe at the coil center when the primary coil is turned off. As is seen in Fig. 3, the magnetic field, or the current of the
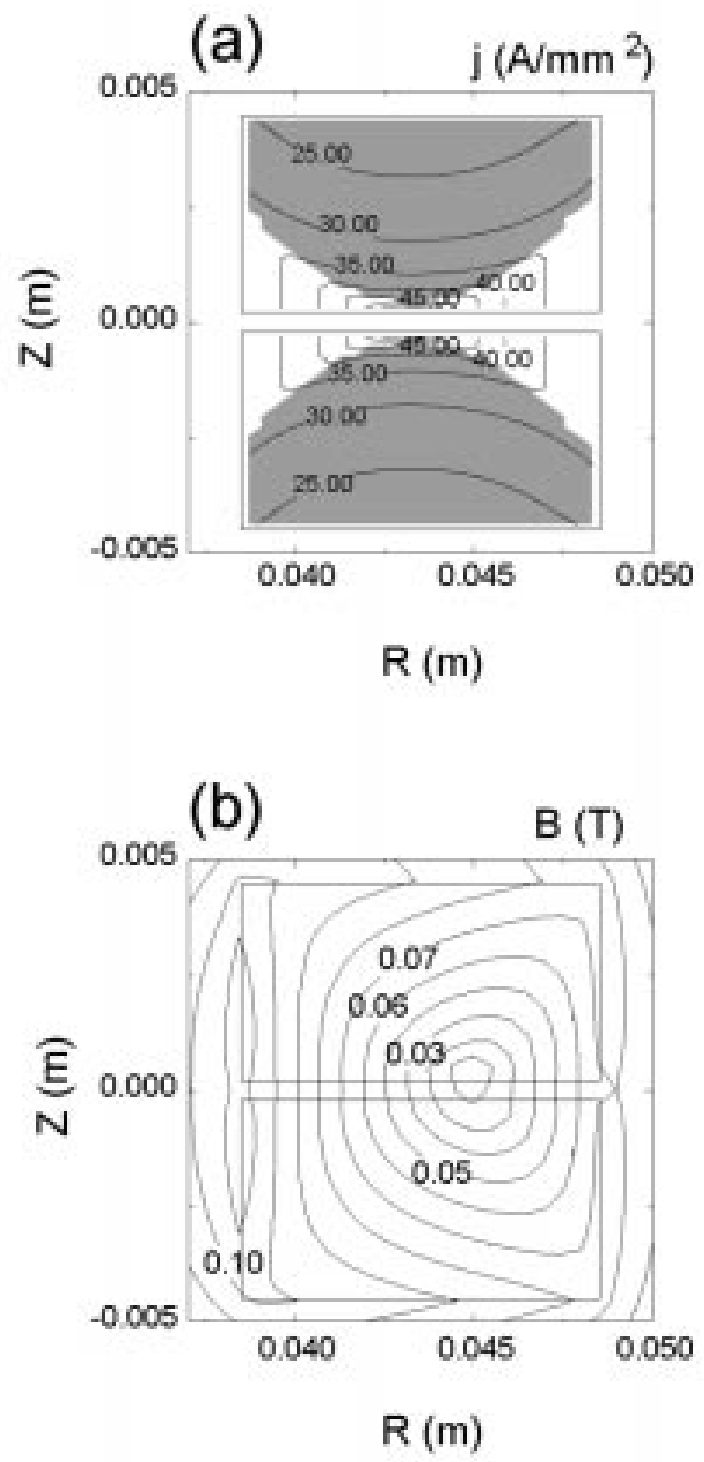

Fig. 2. Spatial profiles of (a) the expected current density and (b) magnetic field when the coil current reaches to the critical condition. There are 22 turns of winding in each block (pancake) starting from the inner radius of $38.5 \mathrm{~mm}$. In (a), the shaded area corresponds to the region where the critical current density is reached.

floating coil, decays rather quickly at the initial phase, however, the decay time becomes longer in the later phase. We consider that the final decay time constant (3306 s) is explained by the joint resistance. From the calculated self-inductance of $0.27 \mathrm{mH}$ of the floating coil, the observed time constant gives a joint resistance of $84 \mathrm{n} \Omega$. On the other hand, the joint resistance was actually measured by preparing a short sample with a soldered lap joint of $50 \mathrm{~mm}$. From this experiment, the joint resistance of the floating coil can be given as $67 \mathrm{n} \Omega$, which is a bit smaller than that estimated from the decay time constant. This discrepancy might be reasonable when considering the difficulty in actually performing the lap joint in the miniature coil.

The fast decay of the magnetic field at the initial phase can be explained by the flux-flow resistance of the HTS tape. As is shown in Fig. 3, a numerical calculation confirms that the 


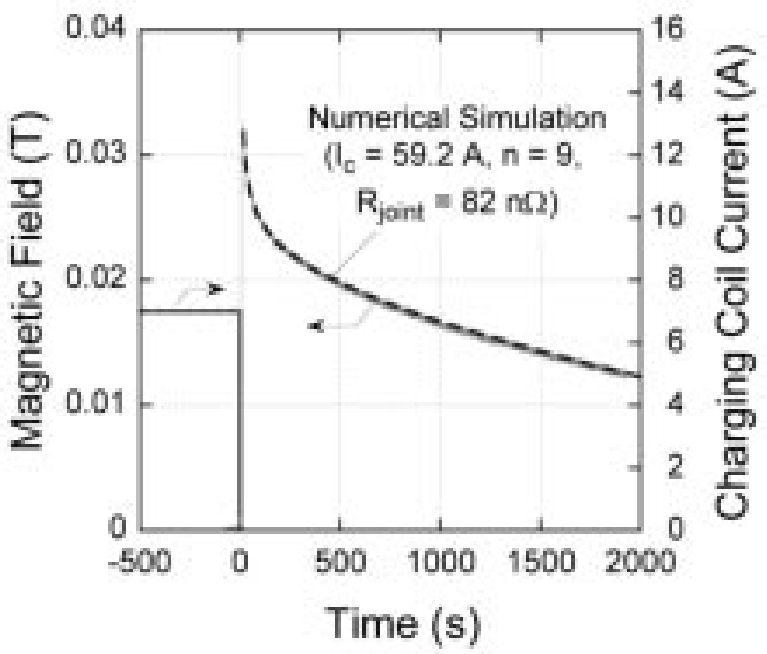

Fig. 3. Waveform of the magnetic field measured by a Hall probe at the center of the FB-RT HTS coil. The measurement started $\sim 12 \mathrm{~s}$ after the turn-off of the current in the primary coil. The coil current is estimated to be $47 \mathrm{~A}$ when the magnetic field is $0.03 \mathrm{~T}$. The dashed line indicates the numerically analyzed waveform.

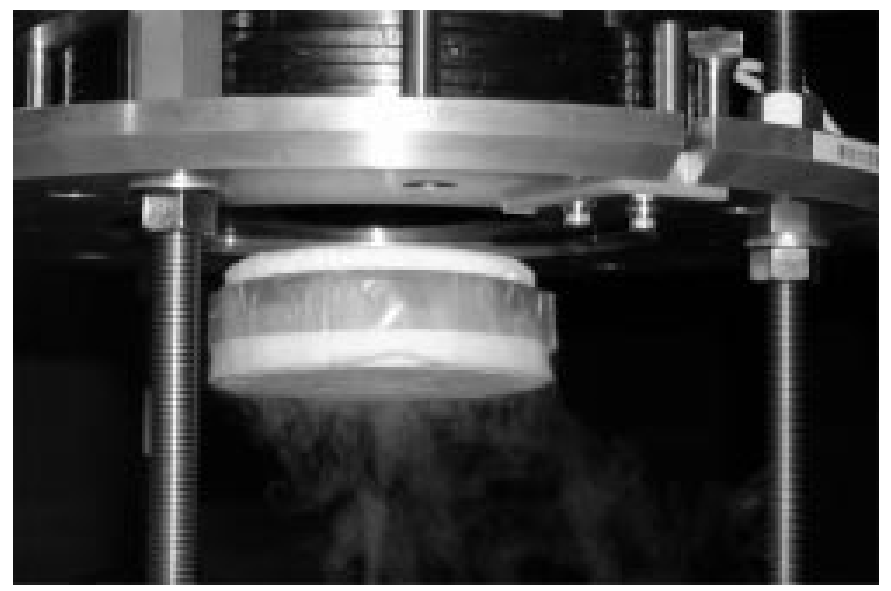

Fig. 4. Photograph of the FB-RT floating coil during magnetic levitation experiments under the copper levitating coil. The bottom surface of the floating coil is irradiated by three laser beams.

measured curve can be well simulated by assuming the $n$-value of 9 for the HTS tape.

Magnetic levitation experiments have been conducted by locating the floating coil at the specified position below the levitating coil made of copper conductors. The feedback control system was activated with laser beam displacement gauges. Three laser beams irradiated the bottom surface of the floating coil and the coil position was evaluated by averaging the three signals. We have observed that magnetic levitation was successfully realized for up to $5 \mathrm{~min}$ in the air. From the laser signals, it was confirmed that the positional accuracy of $\sim 30 \mu \mathrm{m}$ was obtained with the present feedback control.

\section{DEVELOPMENT OF HTS-PCS}

As to the actual floating coil used for the Mini-RT device, a pair of demountable internal electrodes will be installed so that direct excitation will be possible. This will help carry out more flexible plasma experiments than the case of "field-cooling"

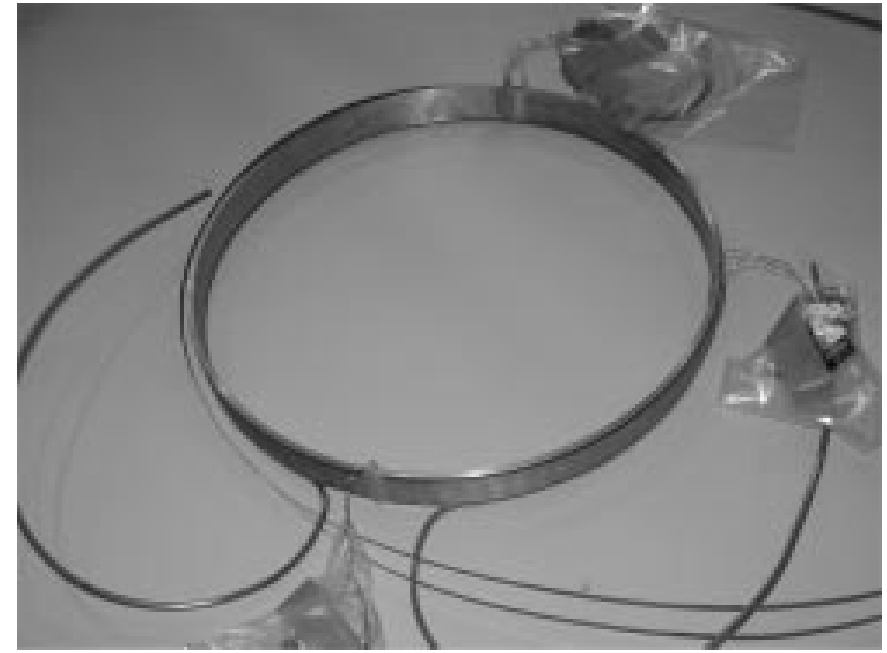

Fig. 5. Photograph of the prototype HTS-PCS developed for the Mini-RT project.

which requires to increase the coil temperature above the critical temperature to restart charging after one cycle of plasma experiment. In this connection, a persistent current switch (PCS) will have to be installed in the Mini-RT floating coil. Since there has not been an example of PCS made of HTS, we need to develop one to be used in this project. One of the most difficult problems related with the development of HTS-PCS is how we can have sufficiently high resistance when the switch is supposed to be turned off. For this purpose, we adopted a Bi-2223 tape conductor with $0.3 \mathrm{wt} \%$ manganese in the silver sheath. By using this conductor, the normal-resistance is estimated to be $0.27 \Omega$ (with a total length of $21 \mathrm{~m}$ ) just above the critical temperature, and this is acceptable for charging the floating coil.

Fig. 5 shows a photograph of the prototype HTS-PCS developed for the Mini-RT project. The HTS tape is wound into a groove of a brass bobbin which has a diameter of $\sim 337 \mathrm{~mm}$. A bifilar winding method was employed for the HTS tape with a $\sim 100 \mathrm{~mm}$ joint at the inner layer, so that the PCS is a noninductive coil which will not affect the magnetic field generated by the floating coil. A Manganin heater is wound at the inner side of the PCS, and this is used to increase the temperature above the critical temperature of the superconductor so that the PCS is turned off.

The experiment of the HTS-PCS has been conducted using a cryostat in which liquid helium was stored at the bottom. Helium gas was supplied from the outside to the PCS through a heat exchanger immersed in liquid helium. An electrical heater was used to control the supply gas temperature. It was confirmed that the PCS can be effectively turned off when the temperature exceeds the critical temperature of $\sim 106 \mathrm{~K}$. We confirmed that the designed resistance of $0.27 \Omega$ could be actually obtained at around the critical temperature.

During the experiment of the HTS-PCS, the voltage versus current curve was measured by changing the temperature from $20 \mathrm{~K}$ to $70 \mathrm{~K}$. Fig. 6 shows an example of the obtained $V-I$ curve when the critical current was measured at $77 \mathrm{~K}$ (cooled by liquid nitrogen in this case). As is seen in Fig. 6 , the $n$-value of the present winding is confirmed to be higher than 12. Fig. 7 shows the measured critical current as a function of the PCS 


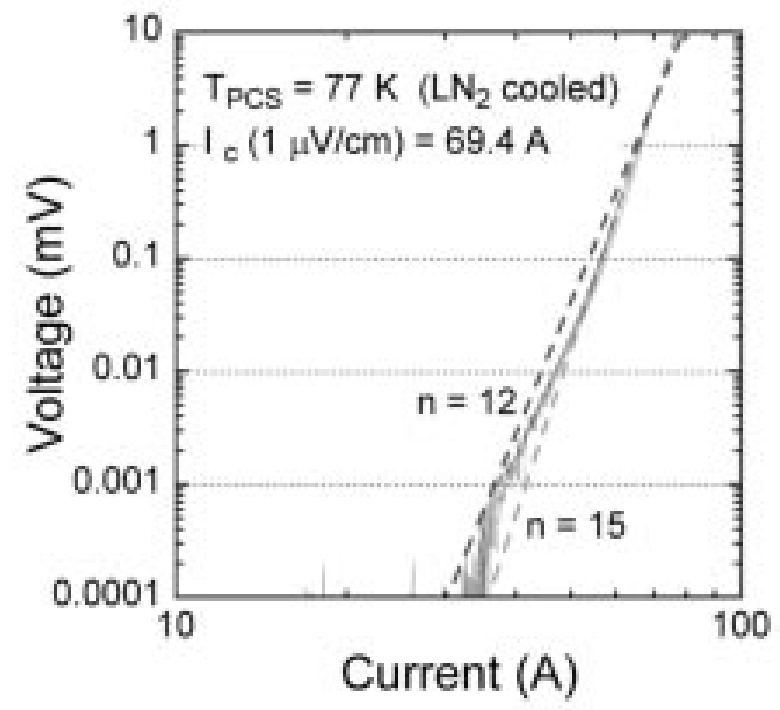

Fig. 6. Voltage versus current curve measured for the HTS-PCS cooled by liquid nitrogen. Two curves determined by the $n$-value of 12 and 15 are indicated by dashed lines.

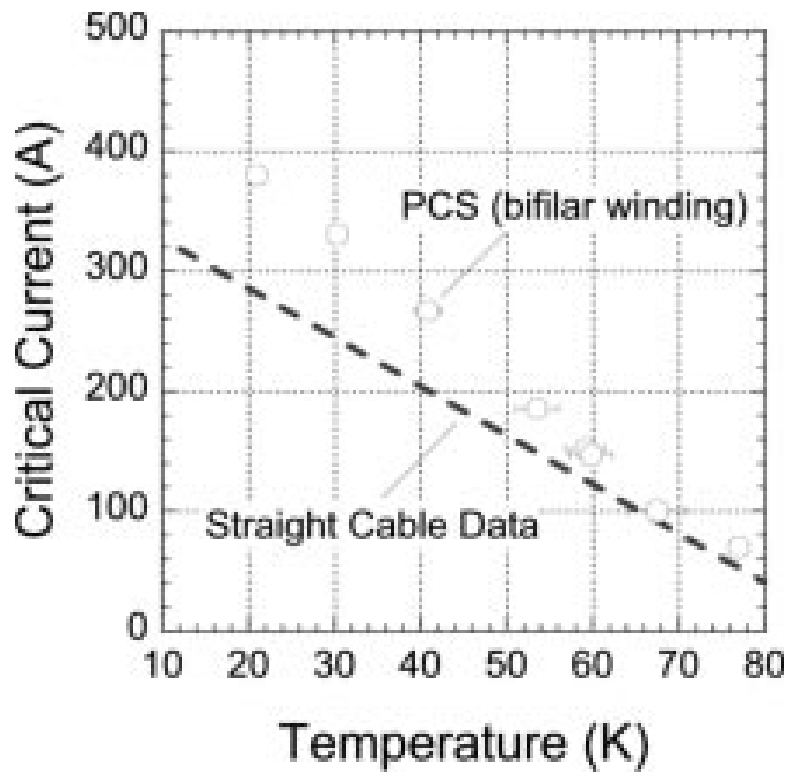

Fig. 7. Dependence of the measured critical current on the PCS temperature. Critical current for a straight sample is deduced for the present conductor based on the empirical scaling given in [6].

temperature. The scatter found in the measured temperature is due to poor thermal insulation of the PCS in the present experiment. As is seen in Fig. 7, the measured critical current seems to be higher than the data given for a straight sample [6]. We consider that a high critical current obtained in the HTS-PCS is due to the fact that the magnetic field in the bifilar winding is much lower than that for a straight sample.
TABLE II

MAJOR SPECIFICATIONS OF HTS-PCS

\begin{tabular}{lc}
\hline Inner Diameter & $337.3 \mathrm{~mm}$ \\
Number of Turns & 20 \\
Winding Method & Bifilar \\
Cable Type & (Ag-0.3wt\%Mn \\
Cable Supplier & sheathed) Bi-2223 \\
Cable Width / Thickness & Sumitomo Electric \\
Silver Ratio & $3.8 / 0.26 \mathrm{~mm}$ \\
Insulation between Turns & 1.9 \\
Cable Length & Formvar \\
Operation Temperature & $21.24 \mathrm{~m}$ \\
Nominal Current (at 20 K) & $20 \mathrm{~K}$ \\
Critical Current (77K, self-field) & $127.6 \mathrm{~A}$ \\
Turn-off Resistance & $53 \mathrm{~A}$ \\
Heater & $0.27 \Omega$ \\
Number of Turns & $\phi 0.5 \mathrm{~mm} \mathrm{Manganin}$ \\
Resistance & 79 \\
Nominal Power & $202.5 \Omega$ \\
\hline
\end{tabular}

\section{SUMMARY}

A magnetically levitated superconducting coil system is being developed using high temperature superconductors for Mini-RT project. A miniature HTS floating coil was fabricated and magnetic levitation experiments have been successfully carried out with a feedback control using laser displacement gauges. A prototype model of a HTS persistent current switch has been fabricated and its characteristics have been confirmed by changing the temperature.

\section{ACKNOWLEDGMENT}

The authors would like to thank Dr. Iwamoto, Mr. Taguchi, and Mr. Tsuge for their support in the experiments of FB-RT and HTS-PCS. They are also grateful for the technical information given by $\mathrm{Dr}$. L. Masur about the Bi-2223 tape used for the FB-RT floating coil.

\section{REFERENCES}

[1] S. M. Mahajan and Z. Yoshida, "Double curl Beltrami flow: Diamagnetic structures," Phys. Rev. Lett., vol. 81, pp. 4863-4866, 1998.

[2] Y. Ogawa, H. Himura, and S. Kondoh et al., "Design of a toroidal plasma confinement device with a levitated superconducting internal coil," in AIP Conf. Proc., 1999, pp. 417-422.

[3] J. H. Schultz, J. Kesner, and J. V. Minervini et al., "The Levitated Dipole Experiment (LDX) magnet system," IEEE Trans. Appl. Supercond., vol. 9, pp. 378-381, 1999.

[4] J. Morikawa, D. Ozawa, and Y. Ogawa et al., "Levitation experiment using a high-temperature superconductor coil for a plasma confinement device," Jpn. J. Appl. Phys., vol. 40, pp. 1029-1031, 2001.

[5] L. Masur, D. Parker, and E. M. Tanner et al., "Long length manufacturing of high performance BSCCO-2223 tape for the Detroit Edison power cable project," IEEE Trans. Appl. Supercond., vol. 11, pp. 3256-3259, 2001.

[6] T. Kaneko, T. Hikata, and M. Ueyama et al., "Status of Bi-2223 tapes performance and development," IEEE Trans. Appl. Supercond., vol. 9, pp. 2465-2468, 1999. 